\title{
Dupuytren's contracture and alcohol
}

\author{
ANTHONY BRADLOW AND ALASTAIR G MOWAT \\ From the Rheumatology Unit, The Nuffield Orthopaedic Centre, Oxford OX3 7LD
}

SUMMARY Reported alcohol consumption was quantified and scored by a validated questionnaire administered by an interviewer to 64 patients (10 female) with Dupuytren's contracture $\vec{\omega}$ (DC) before hand surgery and to 89 controls (44 female) admitted for other hand or foot surgery. Serum urate (SUA), $\gamma$-glutamyl transferase (GGT), and mean red cell volume (MCV) were measured on admission. Thirteen of 54 men with DC reported current daily alcohol intake of 40 get or more compared with one of 45 male controls $(p=0.0001)$. Two of 10 women with DC (butt. none of 44 controls) admitted consuming at least $40 \mathrm{~g}$ alcohol daily $(p=0.03)$. MCV was higher in ${ }_{\perp}$ men (but not women) with DC than in controls $(\mathrm{p}<0.0005)$. Current alcohol consumption scoreo of patients with DC correlated with SUA $(r=0.308, p<0.05), \operatorname{MCV}(r=0.44, p<0.01)$, and GGT $(\mathrm{r}=0.54, \mathrm{p}<<0.001)$ on admission. DC among men is strongly associated with heavy drinking, reflected both in self reporting and haematological data.

Key words: alcohol drinking, erythrocyte indices.

The aetiological factors in Dupuytren's contracture (DC) are uncertain, though genetic, sexual, and racial :nfluences can be implicated to explain its predominance in Caucasian males with a family history of DC. ${ }^{1}$ Links between alcohol consumption and DC have long been recognised. ${ }^{1-7}$ Alcoholic cirrhotics have DC significantly more often than non-cirrhotic controls, ${ }^{2}$ but the frequency of DC in men who are heavy drinkers does not depend on the presence of cirrhosis. ${ }^{5}$ Some degree of liver damage, however, is probably required for the development of DC in heavy drinkers; liver function tests are more often abnormal in heavy drinkers with DC than in those without. ${ }^{6}$ Despite this apparent relationship alcohol intake among patients with DC has been little quantified, ${ }^{8}$ and never using validated modern' methods in a British population. We have compared reported alcohol consumption and the alcohol related haematological and biochemical variables serum urate (SUA), ${ }^{9}$ mean cell volume $(\mathrm{MCV}),{ }^{10}$ and $\gamma$-glutamyl transferase $(\mathrm{GGT})^{11}$ in a group of DC patients requiring surgery and in controls.

\section{Patients and methods}

Sixty four consecutive DC patients (10 female)

Accepted for publication 27 September 1985

Correspondence to Dr Anthony Bradlow. Department of Rheumatology. The Nufficld Orthopaedic Centre, Headington. Oxford OX3 7LD admitted by five consultants to a single orthopaedic unit for fasciectomy or finger amputation werê studied. Mean age of DC patients was 62 years (SD尺 11.2) for males and 63 years (SD 17.7) for females $\vec{F}$ The indication for surgery was a positive table tops test $^{12}$ or gross flexion deformity of the interphal angeal joints of the fifth finger, with hyperextensior at the metacarpophalangeal joint. No assessment was made of the severity of the $D^{13}$ or $o \overleftarrow{E}$ conditions associated with DC such as Peyronie's disease, plantar fascial thickening, or knuckle pads. ${ }^{12}$ Eighty nine control subjects (44 females from the same general population who formed the basis for an earlier survey of alcohol related ortho음 paedic problems ${ }^{14}$ had been electively admitted t\$ the same unit for other surgery to hands or feet? including nail bed ablation, Keller's operation, and toe straightening procedures. Mean age of controls was 59.8 years (SD 11.2) for men and 64.3 years (SDD 9.2) for women.

All patients and controls completed a validate questionnaire administered by an interviewer to provide details of quantity and frequency (QF) oक्ल past and present alcohol consumption. The ques ${ }^{\circ}$ tionnaire was developed in conjunction with the Oxford University Departments of Communits Medicine and Psychiatry. A score was allocated on $\mathbb{P}$ scale 1-9 depending on alcohol intake $;^{14}$ for exam® ple, a score of 1 represented consumption of les\$ than one drink per month and a score of 9 more tham 
100 drinks per week, where a 'drink' is equivalent to a measure of spirits, a glass of wine or sherry, or a half pint of beer (all approximately $10 \mathrm{~g}$ alcohol). Patients and controls with current or maximal lifetime consumption of $40 \mathrm{~g}$ alcohol or more daily (equivalent to a QF score of 7 or higher) were considered to be heavy drinkers. (Maximal lifetime consumption $=$ maximum daily alcohol consumption for five or more consecutive years of the subject's life over the age of 30 , i.e., the highest sustained daily alcohol intake in a subject's adult life.) This level of consumption was chosen as a threshold for heavy drinking because, being slightly lower than most estimates of the daily amount of alcohol required to cause organic damage, ${ }^{15}$ it allows for likely under-reporting of consumption by patients and controls in this study. SUA, GGT, and MCV were measured by standard methods ${ }^{14}$ in patients with DC and in controls on the morning of admission. DC patients were asked whether they were diabetic, anticonvulsant takers, or whether parents or siblings had DC.

\section{S T A T IS T I C S}

The $\chi^{2}$ test, Fisher's exact test, $t$ test, Mann-Whitney $\mathrm{U}$ test, and product-moment correlation coefficients were used where appropriate.

\section{Results}

REPORTED ALCOHOL CONSUMPTION Thirteen of 54 male DC patients reported current daily alcohol intake of $40 \mathrm{~g}$ or more compared with one of 45 male controls $(p=0.0001)$ (relative risk 13.95). Two of 10 female DC patients (but none of 44 female controls) were consuming $40 \mathrm{~g}$ alcohol or more daily $(p=0.03)$ (Fisher's exact test). Twenty eight of 54 male patients (and 13 of 45 controls) had a maximal lifetime alcohol consumption of $40 \mathrm{~g}$ alcohol or more daily $(p<0 \cdot 05)$. Two of 10 DC women (and two of 44 controls) had maximal lifetime alcohol consumption of $40 \mathrm{~g}$ or more daily (NS).

Liver damage due to alcohol occurs at lower levels of consumption in women than in $\operatorname{men}^{16}$ so additional comparison was made between alcohol consumption of DC subjects and controls at a threshold of $20 \mathrm{~g}$ alcohol daily. At this level there were no significant differences in reported consumption between male and female DC subjects and controls.

SUA, GGT, AND MCV ON ADMISSION (Table 1) $\mathrm{MCV}$ on admission in male DC patients was much greater than in controls; however, the difference in MCV between female patients and controls was not significant. Admission MCV was $95 \mathrm{fl}$ or higher in 12
Table $1 S U A, G G T$, and MCV on admission

\begin{tabular}{|c|c|c|c|c|}
\hline & \multicolumn{2}{|l|}{$D C$ patients } & \multicolumn{2}{|l|}{ Controls } \\
\hline & Male & Female & Male & Female \\
\hline $\begin{array}{l}\text { SUA }(\mu \mathrm{mol} / \mathrm{l}) \dagger \\
\text { Mean (SD) } \\
\text { MCV (fl) } \\
\text { Mean (SD) } \\
\text { GGT (IU/l) }\end{array}$ & $\begin{array}{l}n=48 \\
365(84) \\
n=43 \\
94 \cdot 2(4 \cdot 7) \\
n=40 \\
\quad \text { Median } \\
\text { Range }\end{array}$ & $\begin{array}{l}n=7 \\
307 \quad(107) \\
n=9 \\
90 \cdot 4(4 \cdot 2) \\
17 \\
6-151\end{array}$ & $\begin{array}{l}\mathrm{n}=37 \\
368(88) \\
\mathrm{n}=29 \\
88 \cdot 5(5)^{*} \\
\mathrm{n}=78 \\
\text { Median } \\
\text { Range }\end{array}$ & $\begin{array}{l}n=37 \\
305(83) \\
n=40 \\
88 \cdot 3(3 \cdot 6) \\
21 \cdot 5 \\
-196\end{array}$ \\
\hline
\end{tabular}

${ }^{*} \mathrm{p}<0.0005$ compared with male DC.

†SI conversion: SUA $\mu \mathrm{mol} / 1 \times 0 \cdot 01681=\mathrm{mg} /$.

of 43 male DC patients compared with two of 29 controls $(p=0 \cdot 001)$. Seven of these 12 male DC patients reported current or past alcohol consumption of $40 \mathrm{~g}$ or more daily. One was additionally on anticonvulsant therapy and four had MCVs of just $95 \mathrm{fl}$. Only one man with a significantly raised MCV denied past or present alcohol consumption of $40 \mathrm{~g}$ or more daily. SUA and GGT did not differ significantly between the diagnostic/sex groups, but all DC subjects with GGT 30 IU/l or higher reported current or past alcohol consumption of $40 \mathrm{~g}$ or more daily.

CORRELATIONS BETWEEN ALCOHOL INTAKE AND LABORATORY VALUES IN DC PATIENTS (Table 2)

Correlations for these variables were assessed for both male and female DC patients together. MCV, and particularly GGT, correlated well with current QF score. Correlation between SUA and current QF score was weak. We have previously reported lack of correlation between these variables in this control group. ${ }^{14}$

PRESENCE OF OTHER FACTORS ASSOCIATED WITH DC

Fifteen patients (six female) described DC in a sibling or parent. Ten patients had a history both of heavy drinking and of a relative with DC. Four patients took anticonvulsants (two had an MCV above $95 \mathrm{fl}$ ) and another two patients were insulin dependent diabetics.

Table 2 Correlation between reported current alcohol consumption ( $Q F$ score) $S U A, M C V$ and $G G T$ in DC subjects

\begin{tabular}{lll}
\hline SUA $(n=55)$ & $r=0.308$ & $p<0.05$ \\
MCV $(n=52)$ & $r=0.435$ & $p<0.01$ \\
GGT $(n=40)$ & $r=0.543$ & $p<<0.001$
\end{tabular}




\section{Discussion}

Although this study shows a strong association between current heavy drinking and DC in men, alcohol cannot strictly be proved to be a cause or precipitant of DC without matching the occupations, leisure activities, and social class of patients and controls. Nevertheless, the possibility of an aetiological role for alcohol in DC is strengthened by the failure of previous studies to confirm an association between DC and occupation ${ }^{17}{ }^{18}$ and between alcohol consumption and social class. ${ }^{19}$

The less impressive difference in previous consumption of male DC patients and controls may be due to inaccurate recall of past alcohol consumption. There is no reason to suspect that sustained heavy drinking is an effect of DC. The relatively small number of female DC patients may have blunted the significance of differences between their reported alcohol consumption and that of controls; alternatively, women may consistently under-report their alcohol intake or factors other than alcohol may be more strongly associated with DC in women. ${ }^{20}$ Our results do not suggest that DC in women is associated with alcohol consumption levels lower than those in men.

This study probably underestimated alcohol consumption in both DC patients and controls because under-reporting of alcohol consumption in studies of this type is common. ${ }^{21} 22$ The degree of underreporting, however, is likely to be constant in both groups. Alcohol consumption among the population at large with DC may also be much higher because some may not have reached surgery through unfitness associated with alcohol abuse. Conversely, it is conceivable that some DC patients were aware, through discussions with medical advisers or through 'home research', of known links between alcohol and DC. These patients (but not the controls) might have been influenced by this knowledge to exaggerate their reported alcohol consumption. We think this unlikely because no patient reported knowledge of links between DC and alcohol when the purpose of the study was explained to them.

A frequent coexistence of a positive family history and high alcohol consumption in this study suggests the possibility of some synergism between alcohol and a genetic predisposition in causing the expression of DC. ${ }^{57}$ However, both alcohol and a positive family history are common in DC patients: the association may thus simply be coincidence.

The higher MCV among DC males is largely due to alcohol, with anticonvulsant usage having a minor role. The lack of difference in GGT between DC males and controls is, however, surprising in the light of the very significant correlation between current QF score and GGT, and the high reported: alcohol consumption of DC males compared withos controls. A possible explanation is that some DCo subjects have macrocytosis due to dietary folateo deficiency $^{23}$ associated with alcohol consumption which is not heavy enough to cause liver damage and $\frac{\mathbb{Q}}{2}$ increase in GGT.

The correlation between current alcohol consumption score, MCV, and GGT in the DC subjects is in contrast with the lack of correlation between these variables in our controls shown previously. ${ }^{1}$ 尘

Clearly, MCV and GGT correlate with reportec alcohol consumption only in heavier drinkers; in deed in the absence of other causes an individuar with an MCV greater than $98 \mathrm{fl}$ and a GGT over 50 IU/l has a better than $66 \%$ probability of consuming more than $80 \mathrm{~g}$ alcohol daily. ${ }^{24}$ In the present studyo it is noteworthy that in only one case did a raised MCV identity a DC subject who denied heavy drinking; as we have shown previously, ${ }^{14}$ self reports prove generally to be more sensitive than MCV or $\overrightarrow{0}$ GGT, or both, at reflecting heavy drinking.

This study thus confirms clinically and haematol-: ogically that DC in men is associated with heavyo drinking. However, if alcohol precipitates DC thes mechanisms by which it might do so are unknown. The increase in palmar collagen in DC represents repair phenomenon; ${ }^{25}$ Rabinowitz et al ${ }^{26}$ have shown an alteration in the composition of palmar fat of DC patients. They propose that alcohol alters 3 both palmar and liver fat composition by a hypoxic? mechanism; the altered fat could serve as an irritant precipitating a fibrotic repair response in both liver and palmar fascia (Francis $\mathrm{MJO}$, personal communication). These mechanisms remain speculative? but provide an interesting basis for further researchis into DC.

\section{References}

1 Viljanto J A. Dupuytren's contracture: a review. Semir Arthritis Rheum 1973; 111: 155-76.

2 Wolfe S J, Summerskill W H J, Davidson C S. Thickening and contraction of the palmar fascia (Dupuytren's contracture) associated with alcoholism and hepatic cirrhosis. $N$ Engl $J$ MeP 1956; 255: 559-63.

3 Summerskill W H J, Davidson C S, Dible J H, et al. Cirrhosis of the liver. A study of alcoholic and non-alcoholic patients in Boston and London. N Engl J Med 1960; 262: 1-9.

4 Nazari B. Dupuytren's contracture associated with liver dis $\bar{D}$ ease. J Mount Sinai Hosp NY 1966; 33: 69-72.

5 Su C-K. Patek A J. Dupuytren's contracture. Its associationo with alcoholism and cirrhosis. Arch Intern Med 1970; 126. 278-81.

6 Pojer J, Radivojevic M, Williams T F. Dupuytren's disease. It association with abnormal liver function in alcoholism and epilepsy. Arch Intern Med 1972; 129: 561-6.

7 Anonymous. The puzzle of Dupuytren's contracture [Edito rial]. Lancet 1972; ii: $170-1$. 
8 Wegmann T, Geiser W. Die Dupuytrensche Kontraktur der Hand als internistiches Problem: Untersuchungen zur Aetiologie. Helv Med Acta 1964; i: 66-108.

9 Drum D E. Goldman P A. Jankowski C B. Elevation of serum uric acid as a clue to alcohol abuse. Arch Intern Med 1981; 141: 477-9.

$10 \mathrm{Wu}$ A. Chanarin I, Levi A J. Macrocytosis of chronic alcoholism. Lancet 1974; i: 829-31.

11 Rosalki S B. Rau D. Serum gamma-glutamyl transpeptidase activity in alcoholism. Clin Chim Acta 1972; 39: 41-7.

12 Hueston J T. Dupuytren's contracture. Edinburgh and London: Livingstone, 1963: 51-63.

13 Tubiana R. Michou J, Thomine J M. Scheme for the assessment of deformities in Dupuytren's disease. Surg Clin North Am 1968; 48: $979-84$

14 Bradlow A. Mowat A G. Alcohol consumption in arthritic patients: clinical and laboratory studies. Ann Rheum Dis 1985: 44: $163-8$

15 Alcohol and alcoholism. Report of a Special Committee of the Royal College of Psychiatrists. London: Tavistock Publications. 1979: 140.

16 Ashley M J. Olin J S. Harding Le Riche W. Kornaczewski A. Schmidt W. Rankin J. Morbidity in alcoholics. Evidence for accelerated development of physical disease in women. Arch Intern Med 1977: 137: 883-7.
17 Hueston J T. Dupuytren's contracture. Edinburgh and London: Livingstone, 1963: 15.

18 Mikkelsen O A. Dupuytren's disease-the influence of occupation and previous hand injuries. Hand 1978: 10: 1-8.

19 Plant M A. Risk factors in employment. In: Hore B D. Plant M A. eds. Alcohol problems in employment. London: Croom Helm, 1981.

20 Mathews P. Familial Dupuytren's contracture with predominantly female expression. Br J Plast Surg 1979: 32: 120-3.

21 Brody J A. Mills G S. On considering alcohol as a risk factor in specific discases. Am J Epidemiol 1978; 107: 462-6.

22 Armor D. Polich J M. Stambul H. Appendix A: Reliability and validity of self-reported drinking behaviour. In: Alcohol and treatment. New York: Wiley, 1978: 171.

23 Scott J M. Weir D G. Drug-induced megaloblastic change. Clin Haematol 1980: 9: 587-606.

24 Chick J. Kreitman N. Plant M. Mean cell volume and gamma-glutamyl transpeptidase as markers of drinking in working men. Lancet 1981: i: 1249-51.

25 Brickley-Parsons D. Glimcher M J. Smith R J. Albin R. Adams $\mathrm{J} P$. Biochemical changes in the collagen of the palmar fascia in patients with Dupuytren's discasc. J Bone Joint Surg 1981: 63A: 787-97.

26 Rabinowitz J L. Ostermann L. Bora F W, Stacffen J. Lipid composition and de novo lipid biosynthesis of human palmar fat in Dupuytren's disease. Lipids 1983: 18: 371-4. 